
\title{
25 Research Suare \\ The Effect of Carnitine on the Quality of Life and Sleep-Wake Disturbance in Patients With Liver Cirrhosis
}

Hisamitsu Miyaaki ( $\nabla$ miyaaki-hi@umin.ac.jp )

Nagasaki Daigaku Byoin https://orcid.org/0000-0002-8067-4376

Masafumi Haraguchi

Nagasaki Daigaku Byoin

Satoshi Miuma

Nagasaki Daigaku Byoin

Masanori Fukushima

Nagasaki University: Nagasaki Daigaku

Ryu Sasaki

Nagasaki Daigaku Byoin

Yuri Miyazoe

Nagasaki Kouseikai Hospital

Masaya Shigeno

Japan red cross nagasaki gebaku hospital

Kazuhiko Nakao

Nagasaki Daigaku Byoin

Research article

Keywords: L-carnitine, sleep-wake disturbance, quality of life, liver cirrhosis, muscle cramps

Posted Date: October 29th, 2020

DOI: https://doi.org/10.21203/rs.3.rs-97725/v1

License: (c) (1) This work is licensed under a Creative Commons Attribution 4.0 International License.

Read Full License 


\section{Abstract}

Background: Some patients with liver cirrhosis have sleep-wake disturbance and reduced quality of life (QOL). However, the effects of L-carnitine on QOL and sleep-wake disturbance have not been extensively studied. Thus, we aimed to examine the effects of L-carnitine on the sleep quality and QOL in cirrhotic patients.

Methods: We investigated 19 cirrhotic patients (age: 65.0 \pm 10.2 years, male:female=10:9, Child-Pugh score: $6.5 \pm 1.8$ ) treated with 1500 -mg L-carnitine at Nagasaki University Hospital and its associated hospitals. We used questionnaires to evaluate the patients' sleep-wake disturbance and QOL at the time of entry and 8 weeks.

Results: Cirrhosis-related symptom scores (CSSs), Pittsburgh Sleep Quality Index (PSQI) and General Health $(\mathrm{GH})$ of QOL score improved with L-carnitine treatment (CSS: from 11.5 \pm 9.4 to 9.4 $\pm 3.7, \mathrm{p}=0.015$; PSQI: from $9.6 \pm 5.8$ to $7.4 \pm 3.3, p=0.035$; $\mathrm{GH}$ : from $33.5 \pm 8.2$ to $38.3 \pm 9.8, p=0.05)$. L-carnitine treatment reduced PSQI in 12 out of 14 patients with sleep disturbance (PSQI>5). CSS was improved in 12 cases (63\%), and PSQI was improved in 13 cases (68\%). However, L-carnitine treatment did not improve daytime hypersomnolence, as evaluated by the Epworth Sleepiness Scale. None of patients had any adverse events related to the L-carnitine treatment.

Conclusions: L-carnitine is a safe and useful treatment for cirrhotic patients with reduced sleep quality and QOL.

\section{Background}

Liver cirrhosis (LC) is the end stage of chronic liver disease and is a life-threatening hepatic disorder. The advancement in medical technologies, including anti-viral or anti-cancer therapies, has prolonged the survival period of patients with LC.

Patients with LC have various symptoms, such as abdominal fullness, tremor, hepatic encephalopathy, and jaundice, because of liver function failure and portal hypertension. These symptoms could reduce the patients' quality of life (QOL) $(1,2)$. In addition, these symptoms cause sleep-wake disturbances $(2-4)$.

Sleep-wake disturbance is one of the symptoms of hepatic encephalopathy (3-4). Some studies have claimed that lactulose and branched-chain amino acid-enriched supplementation improved sleep-wake abnormalities $(3,5)$.

L-carnitine (L-beta-hydroxy-gamma-N-trimethyl-aminobutyric acid) is an essential cofactor for $\beta$-oxidation of fatty acids and has a major role in lipid metabolism (6). Supplementation with L-carnitine is effective in improving symptoms, such as general fatigue, muscle cramps, and hepatic encephalopathy, in cirrhotic patients (7-9). L-carnitine is expected to improve the QOL and sleep-wake disturbance; however, there are few reports about the effects of L-carnitine on QOL and sleep-wake disturbance. 
In this study, we examined the effects of L-carnitine on QOL and sleep-wake disturbance in patients with LC.

\section{Methods}

Nineteen patients with LC treated with 1500-mg L-carnitine at Nagasaki University Hospital and its associated hospitals were enrolled in this study. The patients had hepatocellular carcinoma (HCC) and were diagnosed with LC based on the clinical and laboratory data or histologic examination findings of liver biopsy specimens. Patients with overt hepatic encephalopathy were excluded in this study. Patients' clinical characteristic are shown in Table 1 . The patients' QOL and sleep quality were assessed by using a questionnaire at the time of entry and at 8 weeks.

Table 1

Patients' clinical characteristics at the time of entry

\begin{tabular}{|c|c|}
\hline Age (years) & $65.0 \pm 10.2$ \\
\hline Sex (male:female) & 10:9 \\
\hline Child-Pugh score & $6.5 \pm 1.8$ \\
\hline Total bilirubin (mg/dl) & $1.15 \pm 0.61$ \\
\hline Albumin $(g / d l)$ & $3.4 \pm 0.72$ \\
\hline BUN (mg/dl) & $23.8 \pm 18.9$ \\
\hline Creatinine $(\mathrm{mg} / \mathrm{dl})$ & $0.84 \pm 0.28$ \\
\hline \multicolumn{2}{|c|}{ Abbreviation: BUN, blood urea nitrogen } \\
\hline
\end{tabular}

This study was conducted in accordance with the provisions of the 1975 Declaration of Helsinki, and the ethics committee at Nagasaki University Hospital approved this study (Nagasaki University Hospital: No. 16082217). Written informed consent was obtained from each participant.

\section{Questionnaires}

We assessed the severity of muscle cramps by using scores of the visual analog scale (VAS). The cirrhosis-related symptom score (CSS) questionnaire, which we developed, was used to evaluate the cirrhotic symptoms (10). The Epworth Sleepiness Scale (ESS) was used to evaluate daytime hypersomnolence (11). Sleep quality was assessed using the Japanese version of the Pittsburgh Sleep Quality Index (PSQI) (12). Health-related quality of life was evaluated using the Japanese 36-Item Short-Form Health Survey (SF-36) [version 2; Medical Outcomes Trust (Hanover, NH, USA), Health Lab (Hanover, NH, USA), QualityMetric (Lincoln, RI, USA), and Shunichi Fukuhara (iHope International; Kyoto, Japan)] (13) 


\section{Statistical analysis}

The paired or non-paired Student's t test was used to assess the continuous data. Correlations were determined using Pearson's linear regression analysis. All the P-values were two-sided, and $\mathrm{P}<0.05$ was considered to indicate a statistically significant difference. All analyses were performed using SPSS Statistics (version 20.0, IBM Corp., Armonk, NY, USA).

\section{Results}

\section{Efficacy of L-carnitine (Table 2)}

Table 2

; The effect of L-Carnitine treatment

\begin{tabular}{|llll|}
\hline & Pre-treatment & After 8 weeks & P value \\
\hline albumin & $3.48 \pm 0.72$ & $3.42 \pm 0.72$ & 0.46 \\
\hline T-bil & $1.15 \pm 0.61$ & $0.95 \pm 0.43$ & 0.05 \\
\hline ammonia & $93.0 \pm 56.6$ & $89.3 \pm 60.4$ & 0.83 \\
\hline CSS & $11.5 \pm 9.4$ & $9.4 \pm 3.7$ & 0.01 \\
\hline PSQI & $9.6 \pm 5.8$ & $7.4 \pm 3.3$ & 0.03 \\
\hline ESS & $5.8 \pm 4.7$ & $4.8 \pm 3.3$ & 0.45 \\
\hline Physical component summary & $28.5 \pm 17.0$ & $32.8 \pm 13.4$ & 0.17 \\
\hline Physical functioning & $27.4 \pm 19.7$ & $31.8 \pm 16.1$ & 0.15 \\
\hline Role limitations due to poor physical health & $28.0 \pm 17.7$ & $36.3 \pm 14.5$ & 0.11 \\
\hline bodily pain & $43.3 \pm 11.7$ & $44.3 \pm 12.6$ & 0.75 \\
\hline general health perception & $33.5 \pm 8.2$ & $38.3 \pm 9.8$ & 0.05 \\
\hline Metal component summary & $50.4 \pm 11.4$ & $49.5 \pm 8.3$ & 0.72 \\
\hline Mental health & $46.8 \pm 13.1$ & $46.4 \pm 9.8$ & 0.86 \\
\hline Role Emotional & $35.0 \pm 15.9$ & $36.7 \pm 16.9$ & 0.69 \\
\hline Social Functioning & $38.3 \pm 14.2$ & $44.8 \pm 11.7$ & 0.14 \\
\hline Vitality & $41.2 \pm 11.3$ & $43.5 \pm 8.1$ & 0.25 \\
\hline Abbreviation; T-bil, Total bilirubin, CSS, cirrhosis-related symptom score, PSQl, Pittsburgh Sleep & \\
\hline Quality Index, ESS, Epworth Sleepiness Scale & & & \\
\hline
\end{tabular}


The VAS score of muscle cramps had reduced significantly from $4.1 \pm 3.1$ to $2.3 \pm 1.8$ by 8 weeks of Lcarnitine treatment $(p=0.004)$. The VAS score of muscle cramps improved in 12 out of 19 cases. CSS and PSQI also reduced with the L-carnitine treatment (CSS: from $11.5 \pm 9.4$ to $9.4 \pm 3.7, p=0.015$; PSQI: from $9.6 \pm 5.8$ to $7.4 \pm 3.3, p=0.035$ ) (Fig. 1A, B). The L-carnitine treatment improved PSQI in 12 out of 14 patients with sleep disturbance (PSQI > 5). CSS was improved in 12 cases, and PSQI was improved in 13 cases. The ESS as well as physical component summary (PCS) and mental component summary (MCS) of SF-36 did not show significant changes after the 8-week L-carnitine treatment. However, owing to the limited number of patients with low PCS scores of $<45(n=16)$, L-carnitine was found to significantly improve the PCS scores (from 23.4. \pm 12.5 to $32.1 \pm 14.5, p=0.017$ ). General health of PCS also improved with the L-carnitine treatment (from $33.5 \pm 8.2$ to $38.3 \pm 9.8, p=0.05$ ).

Significant changes in total bilirubin, albumin, and ammonia levels were not observed.

\section{The correlation of carnitine effect and each clinical factor}

The change in the CSS was significantly correlated with the change in PSQI $(r=0.705, p=0.001)$ (Fig. 2).

Other factors, including age, body mass index, Child-Pugh score, total bilirubin, albumin, and creatinine, at the pre-treatment level were not significantly correlated with the change in PSQI and CSS.

\section{Safety}

None of the patients showed adverse effects caused by the L-carnitine treatment.

\section{Discussion}

This study demonstrated the efficacy of L-carnitine on sleep-wake disturbance and QOL in patients with LC. Some previous studies showed that the prevalence of sleep-wake disturbance was higher in LC patients than in controls $(2,3,14)$. The causes of sleep-wake disturbance are related to hepatic encephalopathy, muscle cramps, melatonin metabolism disturbance, restless legs syndrome, and glucose level fluctuation $(2,3,15-18)$.

Adenosine triphosphate (ATP) reduction, which is as an alteration of energy metabolism, is found in LC patients (19). ATP reduction may cause general fatigue and muscle cramps. Carnitine converts fatty acid into energy in the form of ATP (20). Therefore, L-carnitine administration can improve some symptoms, such as general fatigue and muscle cramps, in cirrhotic patients.

A large Japanese cohort study showed that muscle cramps influence the sleep quality in $37.4 \%$ of patients with chronic liver disease (15). Nakanishi et al. described that an 8-week L-carnitine treatment improved the muscle cramps of patients with LC (9). Therefore, the improvement in muscle cramps can lead to the improvement in sleep quality. 
L-carnitine treatment also significantly improved the VAS score for muscle cramps and CSS in this study. The change in CSS was significantly correlated with the change in PSQI. L-carnitine can improve the patients' sleep quality by improving some physical symptoms associated with LC.

GH of PCS assessed by SF-36 improved with carnitine. Previous studies reported that muscle cramps were associated factor of lower general health (1). Therefore, carnitine effect to $\mathrm{GH}$ is also due to the improvement of muscle cramps.

PCS assessed by SF-36 did not improve with carnitine supplementation. However, owing to the limited number of patients with low PCS of $<45$, L-carnitine significantly improved PCS.

In this study, the patients with PSQI of $\geq 6$, indicating the presence of a sleep disorder, showed greater improvement than those without a sleep disturbance. Patients with higher CSS also tend to show a greater improvement than those with lower CSS. Therefore, L-carnitine tend to be more effective in patients with greater decline in QOL and sleep quality.

Some previous studies have reported that L-carnitine improved hepatic encephalopathy in an animal model $(21,22)$. In fact, L-carnitine treatment reduced the ammonia level and improved hepatic encephalopathy in cirrhotic patients $(7,8)$. Hepatic encephalopathy is associated with daytime hypersomnolence $(2,23)$. Thus, L-carnitine is expected to improve the daytime hypersomnolence; however, in our study, the L-carnitine supplementation did not improve the daytime hypersomnolence in our patients, as evaluated by ESS. In our study, an ESS of $\geq 10$, indicating a significant daytime hypersomnolence, was present in only three out of the 19 patients $(15 \%)$. The small number of cases with daytime hypersomnolence can be associated with this result.

In addition, few reports have investigated the adverse effects of L-carnitine $(8,9)$. In this study, no adverse events were found in any of the patients

This study has some limitations. The present study included a small number of patients and lacked a control group. Large-scale randomized controlled studies are needed in the future to validate the efficacy of this treatment.

\section{Conclusions}

L-carnitine administration can be a useful and safe treatment in cirrhotic patients with sleep disturbance and reduced QOL.

\section{Abbreviations}

QOL, quality of life; CSS, cirrhosis-related symptom scores; PSQI, Pittsburgh Sleep Quality Index; LC, liver cirrhosis; VAS, visual analog scale; ESS, Epworth Sleepiness Scale; PCS, physical component summary ; MCS, mental component summary; ATP; adenosine triphosphate 


\section{Declarations}

\section{Ethics approval and consent to participate}

Written informed consent was obtained from all patients. This study was approved by the ethics committee of Nagasaki University (No. 16082217).

\section{Consent to publish}

Not applicable.

\section{Availability of data and materials}

The datasets during and/or analysed during the current study available from the corresponding author on reasonable request.

\section{Competing interests}

The authors declare that they have no competing interests.

\section{Authors' Contributions}

HM: study concept and design, data collection, data analysis, and writing of the draft manuscript; $\mathrm{MH}$, SM, MF, RS, YM and MS: data collection; and KN: study co-design and review of the manuscript. All authors have approved the final version of the article.

\section{Acknowledgements}

Not applicable.

\section{Funding}

No funding was received.

\section{References}

1. Marchesini G, Bianchi G, Amodio P, Salerno F, Merli M, Panella C, et al.. Factors associated with poor health-related quality of life of patients with cirrhosis. Gastroenterology 2001;120:170-178.

2. Montagnese S, Middleton B, Skene DJ, Morgan MY. Night-time sleep disturbance does not correlate with neuropsychiatric impairment in patients with cirrhosis. Liver Int 2009;29:1372-1382.

3. Ichikawa T, Naota T, Miyaaki H, Miuma S, Isomoto H, Takeshima F, et al. Effect of an oral branched chain amino acid-enriched snack in cirrhotic patients with sleep disturbance. Hepatol Res 2010;40:971-978.

4. Watanabe A. Cerebral changes in hepatic encephalopathy. J Gastroenterol Hepatol 1998;13:752-760. 
5. Singh J, Sharma BC, Puri V, Sachdeva S, Srivastava S. Sleep disturbances in patients of liver cirrhosis with minimal hepatic encephalopathy before and after lactulose therapy. Metab Brain Dis 2017;32:595-605.

6. Tanphaichitr V, Leelahagul P. Carnitine metabolism and human carnitine deficiency. Nutrition 1993;9:246-254.

7. Malaguarnera M, Pistone G, Elvira R, Leotta C, Scarpello L, Liborio R. Effects of L-carnitine in patients with hepatic encephalopathy. World J Gastroenterol 2005;11:7197-7202.

8. Shiraki M, Shimizu M, Moriwaki H, Okita K, Koike K. Carnitine dynamics and their effects on hyperammonemia in cirrhotic Japanese patients. Hepatol Res 2017;47:321-327.

9. Nakanishi H, Kurosaki M, Tsuchiya K, Nakakuki N, Takada H, Matsuda S, et al.. L-carnitine reduces muscle cramps in patients with cirrhosis. Clin Gastroenterol Hepatol 2015;13:1540-1543.

10. Yoshimura E, Ichikawa T, Miyaaki H, Taura N, Miuma S, Shibata $H$, et al. Screening for minimal hepatic encephalopathy in patients with cirrhosis by cirrhosis-related symptoms and a history of overt hepatic encephalopathy. Biomed Rep 2016;5:193-198.

11. Johns MW. A new method for measuring daytime sleepiness: the Epworth sleepiness scale. Sleep 1991;14:540-545.

12. Doi Y, Minowa M, Uchiyama M, Okawa M, Kim K, Shibui K, et al. Psychometric assessment of subjective sleep quality using the Japanese version of the Pittsburgh Sleep Quality Index (PSQI-J) in psychiatric disordered and control subjects. Psychiatry Res 2000;97:165-172.

13. Fukuhara S, Bito S, Green J, Hsiao A, Kurokawa K. Translation, adaptation, and validation of the SF36 Health Survey for use in Japan. J Clin Epidemiol 1998;51:1037-1044.

14. Ghabril M, Jackson M, Gotur R, Weber R, Orman E, Vuppalanchi R, et al. Most individuals with advanced cirrhosis have sleep disturbances, which are associated with poor quality of life. Clin Gastroenterol Hepatol 2017;15:1271-1278.e6.

15. Iwasa M, Karino Y, Kawaguchi T, Nakanishi H, Miyaaki H, Shiraki M, et al. Relationship of muscle cramps to quality of life and sleep disturbance in patients with chronic liver diseases: a nationwide study. Liver Int 2018;38:2309-2316.

16. Montagnese S, Middleton B, Mani AR, Skene DJ, Morgan MY. On the origin and the consequences of circadian abnormalities in patients with cirrhosis. Am J Gastroenterol 2010;105:1773-1781.

17. Matsuzaki T, Ichikawa T, Kondo H, Taura N, Miyaaki H, Isomoto H, et al. Prevalence of restless legs syndrome in Japanese patients with chronic liver disease. Hepatol Res 2012;42:1221-1226.

18. Haraguchi M, Miyaaki $H$, Ichikawa $T$, Shibata $H$, Honda $T$, Ozawa $E$, et al. Glucose fluctuations reduce quality of sleep and of life in patients with liver cirrhosis. Hepatol Int 2017;11:125-131.

19. Mehta SS, Fallon MB. Muscle cramps in liver disease. Clin Gastroenterol Hepatol 2013;11:1385-1391.

20. Mingrone G. Carnitine in type 2 diabetes. Ann N Y Acad Sci 2004;1033:99-107. 
21. Therrien G, Rose C, Butterworth J, Butterworth RF. Protective effect of L-carnitine in ammoniaprecipitated encephalopathy in the portacaval shunted rat. Hepatology 1997;25:551-556.

22. Ratnakumari L, Qureshi IA, Butterworth RF. Effect of L-carnitine on cerebral and hepatic energy metabolites in congenitally hyperammonemic sparse-fur mice and its role during benzoate therapy. Metabolism 1993;42:1039-1046.

23. Montagnese S, De Pittà C, De Rui M, Corrias M, Turco M, Merkel C, et al. Sleep-wake abnormalities in patients with cirrhosis. Hepatology 2014;59:705-712.

\section{Figures}

\section{Figure 1}

A

B

CSS score

$\mathrm{P}=0.015$ PSQI score

$\mathrm{P}=0.035$

25

25

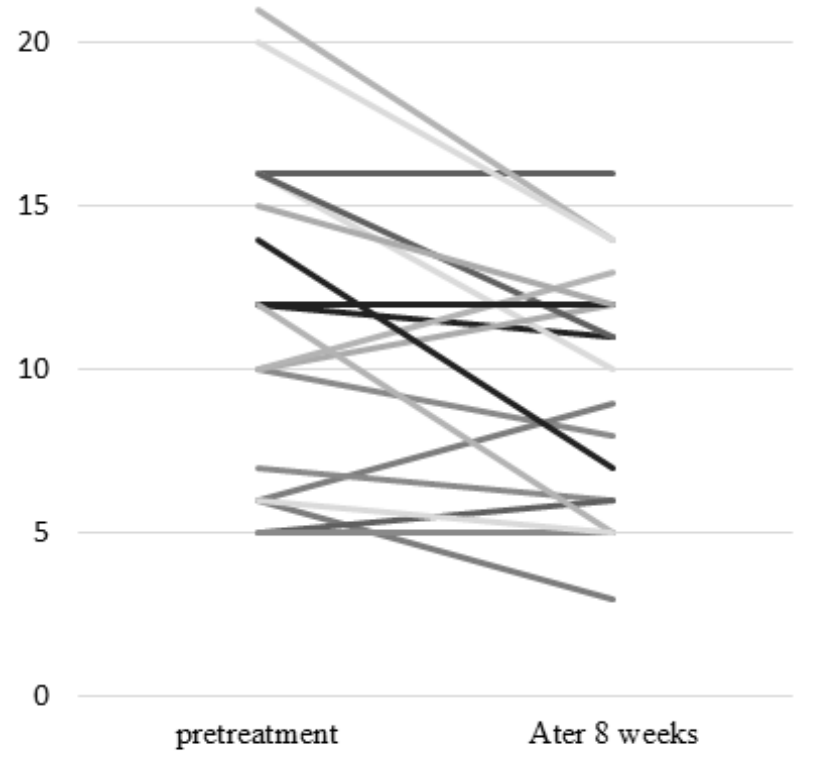

20

15

10

5

0

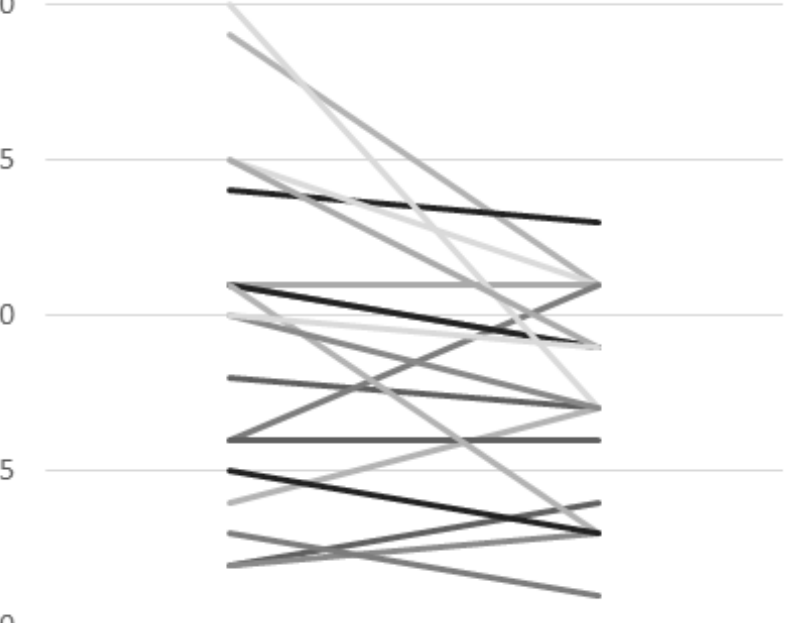

pretreatment

After 8weeks

\section{Figure 1}

(A) The cirrhosis-related symptom score (CSS) before and after the 8-week L-carnitine treatment is shown. The mean CSS improved significantly from $11.5 \pm 9.4$ to $9.4 \pm 3.7$ after the 8-week treatment. (B) 
The Pittsburgh Sleep Quality Index (PSQI) before and after the 8-week carnitine treatment is shown. The mean PSQI decreased significantly from $9.6 \pm 5.8$ to $7.4 \pm 3.3$ after the 8-week treatment.

\section{Figure2}

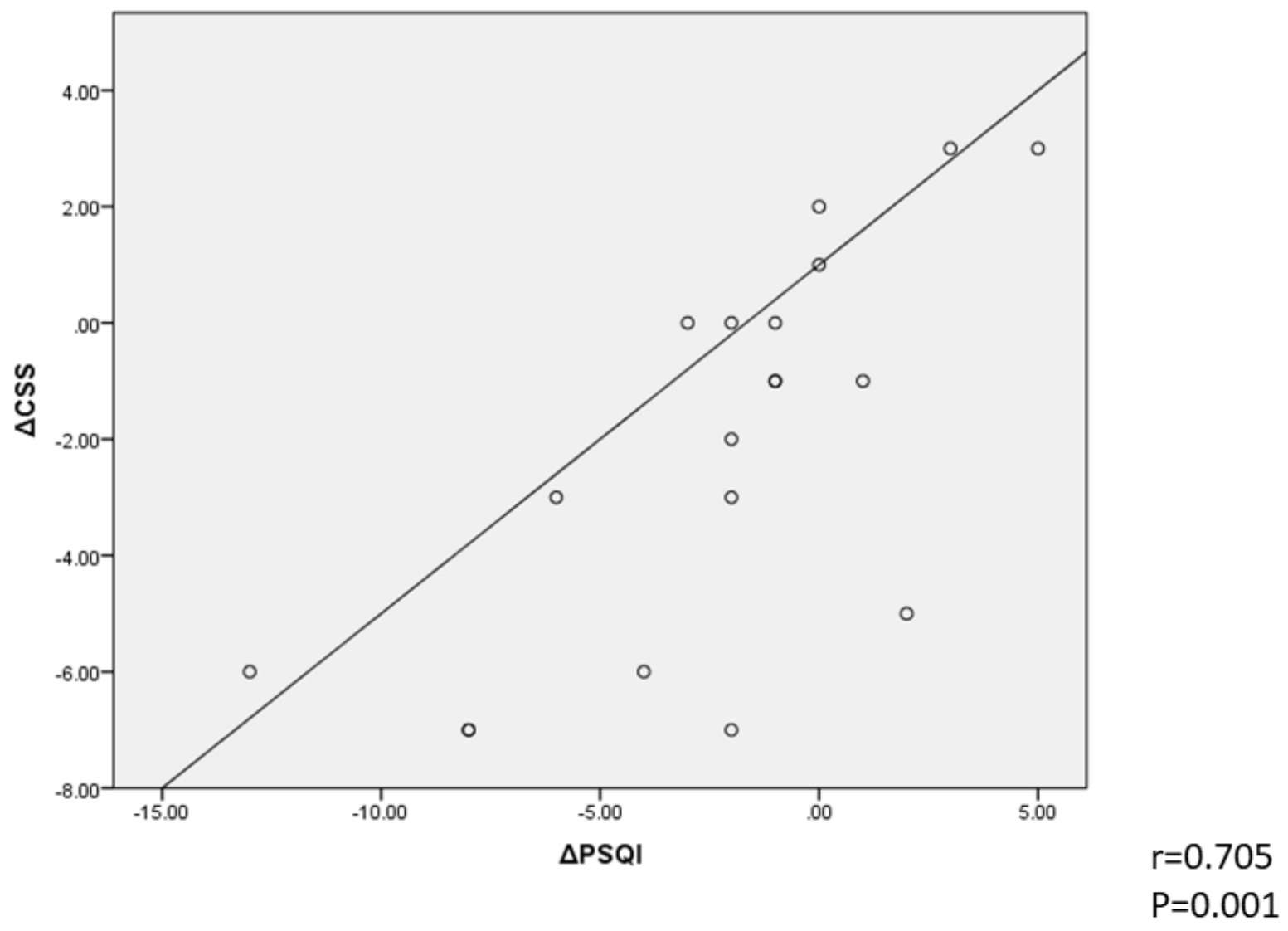

Figure 2

Relationship between the change in cirrhosis-related symptom score (CSS) ( $\triangle \mathrm{CSS}$ ) and change in Pittsburgh Sleep Quality Index (PSQI) ( $\triangle \mathrm{PSQI}) . \triangle \mathrm{CSS}$ showed a significant correlation with $\triangle \mathrm{PSQ}$ $(r=0.705, p=0.001)$. 